\title{
New species and combinations in the pale-spored Agaricales
}

\author{
HARRI HARMAJA
}

\begin{abstract}
HARMAJA, H. 1978: New species and combinations in the pale-spored Agaricales. - Karstenia 18: 29-30.

The following new species are described (all the types are from Finland): Clitocybe gigas Harmaja, Clitocybe subsinopica Harmaja, Cystoderma lilacipes Harmaja, Melanoleuca brevispora Harmaja, and Pleurotus viscidus Harmaja. Clitocybe subdryadicola Harmaja (nomen novum) must replace $C$. serotina Lamoure (non Vel.). The following new combinations are made: Cystoderma jasonis (Cooke \& Massee) Harmaja, Cystoderma terreii (Berk. \& Broome) Harmaja, and Rhodocybe harperi (Murr.) Harmaja.
\end{abstract}

Harri Harmaja, Botanical Museum, University of Helsinki, Unioninkatu 44, SF-00170 Helsinki 17, Finland

Clitocybe gigas Harmaja, n. sp. - A Clitocybe geotropa, quae sat similis est, differt basidiocarpis maioribus (diametro 9-28 cm) et infundibuliformibus, stipite breviore $(4-11 \times 1.2-3.8 \mathrm{~cm})$, lamellis densioribus et angustioribus, hyphis oleiferis lamellarum paucis et sporis sat dissimilibus: $6.5-8.5(-11.0) \times 4.5-6.0(-7.0) \mu \mathrm{m}$, magnitudine variabilibus in eadem lamella, praecipue \pm lacrymoideis sed partim 土 late ellipsoideis. Typus: Finland, prov. Kuusamo, par. Kuusamo, Liikasenvaara, Korvasvaara, alt. $280 \mathrm{~m}$, dolomite area, in litter in luxuriant mixed grass-herb forest and in somewhat drier forest, accompanied by Calypso bulbosa, Carex capillaris, C. digitata, Cypripedium calceolus, Equisetum scirpoides, Fragaria vesca, Poa alpina, Saussurea alpina, Selaginella selaginoides, Rhytidiadelphus triquetrus, Clitocybe lohjaënsis, C. strigosa, Gerronema albidum, Tricholoma bufonium, Helvella hyperborea (on 10.IX.), H. palustris, Lactarius scrobiculatus, Otidea propinquata, 26.VIII.1970 H. Harmaja $(\mathrm{H})$. Other specimens from Kuusamo also seen.

Clitocybe subdryadicola Harmaja, nom. nov. Clitocybe serotina Lamoure, Trav. Sci. Parc Nat. Vanoise 2: 141. 1972; non Clitocybe serotina Vel., Nov. mycol.: 72.1939.
Clitocybe subsinopica Harmaja, n. sp. - Clitocybis sinopicae valde similis, ab ea praecipue differt basidiocarpo typice tenuiore, sporis minoribus $(6.0-8.5 \times 4.0-5.0 \mu \mathrm{m})$ et verisimiliter massa sporarum pallide flavida. Est C. sinopica sensu Bigelowii. - Typus: Finland, prov. Kuusamo, par. Kuusamo, church villace, ca. $5 \mathrm{~km}$ north of church, ca. $500 \mathrm{~m} \mathrm{SE}$ of western end of the small lake Petäjälampi, frequent in site used for German encampment in World War II, in dry heath forest with Pinus sylvestris, 8.VIII.1966 T. Ahti 22794 $(\mathrm{H})$. Specimens also seen from Sweden and the U.S.A.

Cystoderma jasonis (Cooke \& Massee) Harmaja, n. comb. (Agaricus Jasonis Cooke \& Massee in Cooke, Grevillea 16: 77. 1888. Holotype in K examined. - Cystoderma amianthinum var. longisporum Kühn. ex Kühn., Bull. Soc. Linn. Lyon 38: 185. 1969.)

Cystoderma lilacipes Harmaja, n. sp. [Cystoderma longisporum var. purpurascens Heinem. \& Thoen, Bull. Soc. Mycol. France 89: 18. 1973. Not validly published according to 'Code', Art. 43.] - A Cystodermate jasonis differt basidiocarpo tenuiore, pileo brunneo minutissime granuloso, apice stipitis supra zonam annularem violaceo, carne pallida sed non flava, sporis ca. 6.0-7.5 $\times$ 
3.3-4.8 $\mu \mathrm{m}$ (leniter crassioribus). - Typus: Finland, prov. Etelä-Karjala, par. Vehkalahti, NW part of Turkia village, acid rocky outcrops in Larix plantation, on/among Polytrichum juniperinum, 1.X.1977 L. Fagerström (H). Additional specimens from southern Finland also seen.

Cystoderma terreii (Berk. \& Broome) Harmaja, n. comb. (Agaricus Terreii Berk. \& Broome, Ann. Mag. Nat. Hist. 4(6): 462. 1870. Holotype in K examined. - Agaricus granulosus cinnabarinus Secr., Mycogr. suisse 1: 60. 1833. - Cystoderma cinnabarinum (Secr.) Fayod ex Konr. \& Maubl., Icon. Sel. Fung. 3(3): pl. 238. 1927.)

Melanoleuca brevispora Harmaja, n. sp. - Pileus 3-7 cm latus, obscure brunneus. Stipes $6-12 \times$ 0.4-1.2 cm, mediocriter brunneus, glaber. Lamellae albidae. Odor et sapor nullus. Hyphae defibulatae. Sine cystidiis et pileocystidiis. Sporae $5.0-7.5 \times 4.0-5.2 \mu \mathrm{m}$, late ellipsoideae vel subglobosae, tunica verrucosa amyloidea. - Typus: Finland, prov. Varsinais-Suomi, Karkkila (Pyhäjärvi), Haavisto, in deep moss in luxuriant rich fen $500 \mathrm{~m} \mathrm{E}$ of the small lake Iitalampi, with Carex acutiformis, C. appropinquata, C. flava, $C$. heleonastes, Epipactis palustris, Luzula sudetica, Paludella squarrosa, Scorpidium trifarium, Sphagnum warnstorfii, Tomentypnum nitens, Bovista paludosa, 25.IX.1969 H. Harmaja (H).

Pleurotus viscidus Harmaja, n. sp. - Basidiocarpus satis tenuis. Pileus $2-5 \mathrm{~cm}$ latus, leniter et irregulariter hygrophanus, pallide lilacinus dein cum maculis ferrugineis, viscidus. Stipes distinctus, I-2 $\times 0.3-0.8 \mathrm{~cm}$, lateralis vel sublateralis, albus, cum zona annulari. Lamellae albae dein cum maculis ferrugineis, elasticae. Odor et sapor nullus. Hyphae tramatis pilei et lamellarum intricatae, monomiticae, crassitunicatae (usque ad $2.5 \mu \mathrm{m}$ ), fibulatae. Cystidia desunt. Sporae 7.5-10.0 × 2.5-3.5 $\mu \mathrm{m}$, inamyloideae, cylindricae, rectae vel parce curvatae, in massa albae. - Typus: Finland, prov. Kainuu, Kajaani rural commune, Jormua, moist grassherb forest on brookside, in somewhat decayed log of Salix caprea, 25.VIII.1976 H. Harmaja (H).

Rhodocybe harperi (Murr.) Harmaja, n. comb. (Clitocybe Harperi Murrill, Mycologia 5: 209. 1913. Holotype in NY examined.)

Accepted for publication

on April 3, 1978 